\title{
Racial/Ethnic Disparities in Acute Ischemic Stroke Treatment Within a Telestroke Network
}

\author{
Shaun Ajinkya, MD, MPH, ${ }^{1}$ Eyad Almallouhi, $M D_{,}{ }^{1}$ \\ Nancy Turner, RN, BSN, ${ }^{1}$ Sami Al Kasab, MD, ${ }^{2}$ \\ and Christine $A$. Holmstedt, $D O^{1}$ \\ ${ }^{1}$ Department of Neurology, Medical University of South Carolina, \\ Charleston, South Carolina, USA. \\ ${ }^{2}$ Department of Neurology, University of lowa, lowa City, lowa, \\ USA.
}

\begin{abstract}
Background: The growth of telestroke services expanded the reach of acute stroke treatment. However, ethnic disparities in receiving such treatment have not been fully assessed.

Materials and Methods: We reviewed prospectively maintained data on patients evaluated through the Medical University of South Carolina telestroke program between January 2016 and November 2018. Outcomes included odds of receiving intravenous recombinant tissue plasminogen activator ( $t P A)$, receiving mechanical thrombectomy (MT), and achieving door-to-needle (DTN) time $\leq 60$ and $\leq 45 \mathrm{~min}$ among patients receiving $t P A$. We used logistic regression to analyze the contribution of race/ ethnicity.

Results: We included 2,977 patients, of whom 1,093 (36.7\%) identified as nonwhite; of these, 1,048 patients (95.9\%) identified as black or African American. Significantly more nonwhite patients were seen at a primary stroke center (PSC) (68.4\% vs. $52.3 \%$ in whites, $p<0.001)$. However, white patients had significantly higher odds of receiving tPA (odds ratio [OR] 1.47, confidence interval [95\% CI] 1.17-1.84). There was no significant difference in receiving MT between races. Among patients receiving $t P A$, whites had higher odds of DTN $\leq 45 \mathrm{~min}$ (OR 1.76, 1.20-2.57) and $\leq 60 \mathrm{~min}$ (OR 1.87, 95\% CI 1.31-2.66).

Conclusions: White patients had better odds achieving DTN $\leq 45 \mathrm{~min}$ and $D T N \leq 60 \mathrm{~min}$ if receiving $t P A$ within a telestroke setting, as well as higher odds of receiving tPA, even after adjustment for comorbidities. This was noted despite white patients having less access to PSCs. However, larger scale studies are needed to further study the impact of ethnic disparities.
\end{abstract}

Keywords: telemedicine, telehealth, teleneurology, telestroke, emergency medicine, teletrauma

\section{Introduction}

$\mathrm{P}$

revious studies have reported that nonwhite patients, particularly African Americans, experience disparities in stroke care and outcomes in the United States, which lead to differences in terms of both incidence and mortality. ${ }^{1-4}$ Some of these disparities have been attributed, in part, to differentials in access to care. ${ }^{4,5}$ With the expansion of telestroke networks, more hospitals are now capable of administering intravenous recombinant tissue plasminogen activator (tPA). ${ }^{6-8}$ Under the telestroke system of care, patients presenting to community hospitals (spokes) with stroke-like symptoms are evaluated by a stroke expert for eligibility for tPA, and are then transferred to a thrombectomy-capable center (hub) for mechanical thrombectomy (MT) evaluation if deemed candidates for such treatment. ${ }^{9}$ Prior research on ethnic disparities in a telestroke setting is sparse; one study in Texas suggested no differences in access to telestroke, but did not comment on door-to-needle (DTN) times, differences in tPA administration, or MT evaluation. ${ }^{10}$ We aim to determine whether disparities in receiving tPA, receiving MT, and DTN time persist between white and nonwhite patients within a telestroke setting.

\section{Materials and Methods}

\section{SETTING}

We conducted a retrospective chart review of the prospectively collected registry data for all patients seen through the Medical University of South Carolina (MUSC) spoke-and-hub telestroke network. Study duration was between January 2016 and November 2018. The study was approved by the MUSC Institutional Review Board.

\section{DATA COLLECTION}

We included all patients with complete data sets who presented with stroke-like symptoms and were evaluated through video telestroke consultation at a spoke hospital during the study period. Demographic data collected included age, race/ ethnicity, gender, history of hypertension, diabetes, coronary artery disease, stroke, hyperlipidemia, atrial fibrillation, National Institute of Health stroke scale (NIHSS) on admission, and whether the patient was transferred to another hospital. 
Race/ethnicity options collected included white, black, or African American, Hispanic, Asian, or Other. For the purposes of analysis, race was dichotomized as white and nonwhite. We collected data on whether each center was a primary stroke center (PSC), which was defined by accreditation by the Joint Commission. We additionally collected data on several quality measures, including the times in minutes from stroke symptom onset to emergency department (ED) arrival, ED arrival to telestroke page, telestroke page to neurology consultant login, consultant login to tPA decision, tPA decision to tPA administration, and DTN. Patients with missing data in the aforementioned variables were removed.

\section{OUTCOMES}

Our outcomes of interest included odds of receiving tPA and odds of receiving MT. We also investigated the odds of achieving DTNs $\leq 60$ and $\leq 45 \mathrm{~min}$ among patients receiving tPA, the quality benchmarks recommended by the American Heart Association/American Stroke Association (AHA/ASA). ${ }^{11}$

\section{STATISTICAL ANALYSIS}

Descriptive statistics were used to describe patient demographic and clinical characteristics. The Wilcoxon rank-sum test was used to compare median times between different groups if the data were non-normal. Otherwise, we compared the differences between the groups using $t$-tests and chisquare for continuous and categorical variables, respectively. Logistic regression analyses were used to analyze the associations between race and the specified dichotomous outcomes described previously. Covariates considered for the models included age, gender, NIHSS, history of hypertension, diabetes, coronary artery disease, prior stroke, hyperlipidemia, atrial fibrillation, transfer to outside hospital, evaluation at a PSC, and symptom onset to ED door time. All predictors were introduced into the model, whereas final models were generated through backward selection with a $p$-value level of removal at $p<0.2$. All models were checked for collinearity. The predictor of white race was forced into the model. An alpha level of 0.05 was used for the logistic regression analyses; for comparisons of demographics and quality measure times, a Bonferronicorrected alpha level of 0.0025 was used. All analyses were performed by Stata 11 (StataCorp LLC, College Station, TX).

\section{Results}

A total of 2,977 patients were included in these analyses, of whom 1,093 (36.7\%) identified as nonwhite; of these, 1,048 (95.9\%) identified as black or African American. Table 1 displays demographic and clinical characteristics of white and nonwhite patients. White patients had significantly higher

\begin{tabular}{|c|c|c|c|}
\hline & WHITE PATIENTS $(n=1,884)$ & NONWHITE PATIENTS $(n=1,093)$ & $p$ \\
\hline Age, mean (SD) & $67.3(15.1)$ & $60.4(15.8)$ & $<0.001$ \\
\hline Female gender & $1,005(53.3)$ & $653(59.7)$ & 0.001 \\
\hline NIHSS, median (IQR) & $3(1-8)$ & $5(2-10)$ & $<0.001$ \\
\hline History of hypertension & $1,270(67.4)$ & $875(80.1)$ & $<0.001$ \\
\hline History of diabetes & $553(29.4)$ & $473(43.3)$ & $<0.001$ \\
\hline History of coronary artery disease & $328(17.4)$ & $124(11.3)$ & $<0.001$ \\
\hline History of stroke & $467(24.8)$ & $346(31.7)$ & $<0.001$ \\
\hline History of hyperlipidemia & $561(29.8)$ & $287(26.3)$ & 0.04 \\
\hline History of atrial fibrillation & $287(15.2)$ & $68(6.2)$ & $<0.001$ \\
\hline Was tPA given? & $448(23.8)$ & $239(21.9)$ & 0.23 \\
\hline Was thrombectomy performed? & $80(7.9)$ & $39(7.7)$ & 0.90 \\
\hline Symptom onset to ED door, min, median (IQR) & $88.5(45-212)$ & $90(45-226)$ & 0.78 \\
\hline Transferred? & 406 (21.6) & $275(25.2)$ & 0.024 \\
\hline Seen at primary stroke center? & $985(52.3)$ & $748(68.4)$ & $<0.001$ \\
\hline
\end{tabular}

Bold values indicate significance at the Bonferroni-corrected level of $p<0.0025$

ED, emergency department; IQR, interquartile range; NIHSS, National Institutes of Health Stroke Scale; SD, standard deviation; tPA, intravenous recombinant tissue plasminogen activator. 


\begin{tabular}{|c|c|c|c|}
\hline Door-to-telestroke page, median (IQR) & $13(7-23)$ & $18(11-28)$ & $<0.001$ \\
\hline Telestroke page-to-consultant log-in, median (IQR) & $4(2-5.5)$ & $4(2-5)$ & 0.38 \\
\hline Consultant log-in-to-tPA decision, median (IQR) & $17(13-23)$ & $19(15-28)$ & 0.004 \\
\hline DTN, median (IOR) & $49(37-65)$ & $58(44-79)$ & $<0.001$ \\
\hline OTN, median (IQR) & $119(84-160.5)$ & $129(95-172)$ & 0.03 \\
\hline
\end{tabular}

Bold values indicate significance at the Bonferroni-corrected level of $p<0.0025$.

DTN, door-to-needle; OTN, symptom onset to needle.

mean age (67.3 vs. 60.4 in nonwhites, $p<0.001$ ), prevalence of coronary artery disease $(17.4 \%$ vs. $11.3 \%$ in nonwhites, $p<0.001)$, and atrial fibrillation $(15.2 \%$ vs. $6.2 \%, p<0.001)$. Nonwhite patients were significantly more female $(59.7 \%$ vs. 53.3\%, $p=0.001$ ), had higher median admission NIHSS (5 interquartile range [IQR 2-10] vs. 3 [IQR 1-8] in whites, $p<0.001$ ), and higher baseline prevalence of hypertension (80.1\% vs. $67.4 \%$ in whites, $p<0.001)$, diabetes $(43.3 \%$ vs. $29.4 \%$ in whites, $p<0.001$ ), and prior stroke ( $31.7 \%$ vs. $24.8 \%$, $p<0.001)$. Significantly more nonwhite patients were seen at a PSC (68.4\% vs. $52.3 \%$ in whites, $p<0.001)$. There was no significant difference between unadjusted levels of tPA administration or MT performance between races over telestroke.

Table 2 gives time metrics in patients receiving tPA. African Americans had significantly higher door to telestroke page (median 18 vs. $12 \mathrm{~min}, p<0.001$ ) time, tPA decision to tPA administration time (median 12 vs. $9 \mathrm{~min}, p<0.001$ ), and DTN (median 58 vs. $49 \mathrm{~min}, p<0.001$ ).

Table 3. Adjusted Odds Ratios for Intravenous Recombinant Tissue Plasminogen Activator Administration in All Patients

\begin{tabular}{l|c}
\multicolumn{1}{c|}{ PREDICTOR } & OR (95\% CI) \\
White race & $1.47(1.17-1.84)$ \\
\hline NIHSS (1-U increment) & $1.04(1.03-1.06)$ \\
\hline History of atrial fibrillation & $0.58(0.41-0.81)$ \\
\hline History of stroke & $0.51(0.40-0.66)$ \\
\hline Seen at primary stroke center & $1.54(1.23-1.92)$ \\
\hline Transferred to outside hospital & $8.39(6.48-10.9)$ \\
\hline Symptom onset to ED arrival (1-min increment) & $0.99(0.99-0.99)$ \\
\hline
\end{tabular}

Bold values indicate significance at the level of $p<0.05$.

$\mathrm{Cl}$, confidence interval; $\mathrm{OR}$, odds ratio.
Table 3 displays the adjusted model predictors for receiving tPA. White patients had significantly higher odds of receiving tPA after adjusting for admission NIHSS, history of atrial fibrillation, history of stroke, being seen at a PSC, transfer to outside hospital, and symptom onset to ED door time (odds ratio [OR] 1.47, confidence interval [95\% CI] 1.17-1.84). White race was not significantly associated with higher odds of thrombectomy (OR 1.44, 95\% CI 0.92-2.25) (Table 4).

Among patients who received tPA, we found that white patients had significantly higher odds of achieving a DTN $\leq 45 \mathrm{~min}$, after adjusting for age, gender, NIHSS, hypertension, coronary artery disease, prior stroke, thrombectomy performance, evaluation at a PSC, and transfer to outside hospital (OR 1.76, 1.20-2.57). Similarly, white patients had higher odds of achieving DTN $\leq 60 \mathrm{~min}$ after adjustment for age, gender, NIHSS, history of coronary artery disease, symptom onset to ED door time, and transfer to an outside hospital (OR 1.87, 95\% CI 1.31-2.66) (Tables 5 and 6).

\section{Table 4. Adjusted Odds Ratios for Thrombectomy} in All Patients

\begin{tabular}{l|c}
\multicolumn{1}{c|}{ PREDICTOR } & OR (95\% Cl) \\
\hline White race & $1.44(0.92-2.25)$ \\
\hline NIHSS (1-U increment) & $1.04(1.02-1.07)$ \\
\hline Female gender & $1.31(0.87-1.99)$ \\
\hline History of hypertension & $0.62(0.39-1.00)$ \\
\hline History of atrial fibrillation & $1.89(1.17-3.09)$ \\
\hline History of stroke & $0.48(0.26-0.88)$ \\
\hline History of hyperlipidemia & $1.58(0.99-2.54)$ \\
\hline Transfer to outside hospital & $380.6(52.1-2776.5)$ \\
\hline Seen at primary stroke center & $1.97(1.30-3.01)$ \\
\hline
\end{tabular}

Bold values indicate significance at the level of $p<0.05$. 
Table 5. Adjusted Odds Ratios for Door-to-Needle Time $\leq \mathbf{4 5} \mathrm{min}$ in Final Model for Patients Receiving Intravenous Recombinant Tissue Plasminogen Activator

\begin{tabular}{l|c}
\multicolumn{1}{c|}{ PREDICTOR } & OR (95\% CI) \\
\hline White race & $1.76(1.20-2.57)$ \\
\hline Age (1-year increment) & $1.01(0.99-1.02)$ \\
\hline NIHSS (1-U increment) & $1.04(1.01-1.07)$ \\
\hline Female gender & $0.72(0.52-1.01)$ \\
\hline History of hypertension & $0.61(0.42-0.89)$ \\
\hline History of stroke & $0.75(0.49-1.16)$ \\
\hline History of coronary artery disease & $0.52(0.32-0.85)$ \\
\hline Thrombectomy performed & $1.70(0.94-3.06)$ \\
\hline Transferred to outside hospital & $0.62(0.41-0.93)$ \\
\hline Seen at primary stroke center & $0.77(0.54-1.10)$ \\
\hline
\end{tabular}

Bold values indicate significance at the level of $p<0.05$.

\section{Discussion}

This study evaluates racial and ethnic disparities in access to stroke treatment and quality measures in telestroke setting. Our study demonstrates that white patients had higher odds of receiving $\mathrm{PPA}$, and that white patients had significantly higher odds of receiving tPA within the recommended time benchmarks recommended by the AHA/ASA.

In this study, we found that a significantly higher percentage of nonwhite patients (of whom our sample was largely African American) were seen at a PSC. This is concordant with findings that overall, a larger percentage of nonwhites have access to a PSC within 60 min. ${ }^{10,12}$

Table 6. Adjusted Odds Ratios for Door-to-Needle Time $\leq \mathbf{6 0} \mathrm{min}$ in Final Model for Patients Receiving Intravenous Recombinant Tissue Plasminogen Activator

PREDICTOR

\begin{tabular}{l|c}
\hline White race & $1.87(1.31-2.66)$ \\
\hline Age (1-year increment) & $1.01(0.99-1.02)$ \\
\hline Female gender & $0.67(0.47-0.93)$ \\
\hline NIHSS (1-U increment) & $1.02(1.00-1.05)$ \\
\hline History of coronary artery disease & $0.56(0.36-0.89)$ \\
\hline Transferred to outside hospital & $0.79(0.55-1.13)$ \\
\hline Symptom onset to ED arrival (1-min increment) & $1.00(0.99-1.00)$ \\
\hline
\end{tabular}

Bold values indicate significance at the level of $p<0.05$.
Interestingly, despite less access to PSC, white patients had higher adjusted odds of receiving tPA even after adjusting for comorbidities and evaluation at a PSC. Furthermore, in our study, we found that white patients had significantly shorter DTN times. The finding of lower tPA use has been shown in African American patients in the past despite adjustment for eligibility. ${ }^{13,14}$ Prior research has indicated that part of the reason for differences in IPA administration between African Americans and whites is due to slower presentation to the hospital. ${ }^{1,15}$ However, this persisted despite ethnic groups having a similar symptom onset to ED arrival time in our study. We additionally found no difference between races in odds of receiving thrombectomy. This contrasts with an analysis of the 2008 Nationwide Inpatient Sample database, wherein it was found that white, Hispanic, and Asian/Pacific Islander patients received endovascular treatment at higher rates than black patients. ${ }^{16}$

To our knowledge, this is the first study analyzing racial/ ethnic differences in various components of DTN among tPA recipients. An overall longer DTN was observed, which appears to be partly driven by the subcomponent of the time between a patient's arrival at the "spoke" hospital and page to the telestroke service. This would imply that there may be differences in how African American patients are evaluated by spoke hospitals. There is well-described literature suggesting that African American patients may have delays in receipt of initial head $\mathrm{CT}^{17,18}$ in the evaluation of acute ischemic stroke, and that, in a general sense, symptoms such as pain are taken less seriously in African American patients. ${ }^{19} \mathrm{It}$ should further be noted that in contrast to other studies suggesting lower 911 use among African Americans in a stroke setting, ${ }^{17}$ it appears that in South Carolina, significantly more African Americans seen over a telestroke network called 911 compared with Caucasians, which may be related to perception of stroke severity, access to transportation, or lack of insurance. $^{4}$

Our study had several limitations. The main limitation is derived from the retrospective and observational nature of the study; there may be unanticipated covariates for which we cannot adjust. In particular, we had a paucity of data on income, insurance status, and stroke subtype, and were thus unable to adjust for this. Moveover, our telestroke sample, as with any large-scale registry, suffered from missing data, which may introduce bias. In addition, our sample had a paucity of Hispanic and Asian American patients, and our results are less generalizable to these groups.

In conclusion, we found that white patients had better odds of achieving a DTN $\leq 45 \mathrm{~min}$ and a DTN $\leq 60 \mathrm{~min}$ if receiving tPA within a telestroke setting, as well as higher odds of 
receiving tPA. Larger scale studies are needed to study the impact of ethnic disparities.

\section{Disclosure Statement}

No competing financial interests exist.

\section{Funding Information}

No funding received for this study.

\section{REFERENCES}

1. Bhattacharya P, Mada F, Salowich-Palm L, et al. Are racial disparities in stroke care still prevalent in certified stroke centers? J Stroke Cerebrovasc Dis 2013; 22:383-388.

2. Boan $A D$, Feng $W W$, Ovbiagele $B$, et al. Persistent racial disparity in stroke hospitalization and economic impact in young adults in the buckle of stroke belt. Stroke 2014;45:1932-1938.

3. Burns SP, White BM, Magwood G, et al. Racial and ethnic disparities in stroke outcomes: A scoping review of post-stroke disability assessment tools. Disabil Rehabil 2019;41:1835-1845.

4. Malek AM, Adams RJ, Debenham E, et al. Patient awareness and perception of stroke symptoms and the use of 911. J Stroke Cerebrovasc Dis 2014; 23:2362-2371.

5. Kimball MM, Neal D, Waters MF, Hoh BL. Race and income disparity in ischemic stroke care: Nationwide inpatient sample database, 2002 to 2008. J Stroke Cerebrovasc Dis 2014;23:17-24.

6. Zhang $D$, Shi L, Ido Moges $S$, et al. Impact of participation in a telestroke network on clinical outcomes. Circ Cardiovasc Qual Outcomes 2019;12: e005147.

7. Al Kasab S, Adams RJ, Debenham E, Jones DJ, Holmstedt CA. Medical University of South Carolina Telestroke: A telemedicine facilitated network for stroke treatment in South Carolina-A progress report. Telemed J E Health 2017; 23:674-677.

8. Almallouhi $\mathrm{E}, \mathrm{Al}$ Kasab S, Harvey JB, et al. Impact of treatment time on the long-term outcome of stroke patients treated with mechanical thrombectomy. J Stroke Cerebrovasc Dis 2019;28:185-190.

9. Powers WJ, Rabinstein AA, Ackerson T, et al. 2018 Guidelines for the Early Management of Patients With Acute Ischemic Stroke: A Guideline for Healthcare Professionals From the American Heart Association/American Stroke Association. Stroke 2018;49:e46-e110.

10. Lyerly MJ, Wu TC, Mullen MT, et al. The effects of telemedicine on racial and ethnic disparities in access to acute stroke care. J Telemed Telecare 2016;22:114-120.

11. Xian $Y, X u H$, Lytle $B$, et al. Use of strategies to improve door-to-needle times with tissue-type plasminogen activator in acute ischemic stroke in clinical practice: Findings from target: Stroke. Circ Cardiovasc Qual Outcomes 2017;10:e003227.

12. Mullen MT, Wiebe DJ, Bowman A, et al. Disparities in accessibility of certified primary stroke centers. Stroke 2014;45:3381-3388.

13. Nasr DM, Brinjikji W, Cloft HJ, Rabinstein AA. Racial and ethnic disparities in the use of intravenous recombinant tissue plasminogen activator and outcomes for acute ischemic stroke. J Stroke Cerebrovasc Dis 2013;22:154-160.

14. Johnston SC, Fung $L H$, Gillum LA, et al. Utilization of intravenous tissue-type plasminogen activator for ischemic stroke at academic medical centers: The influence of ethnicity. Stroke 2001;32:1061-1068.

15. Hsia AW, Edwards DF, Morgenstern LB, et al. Racial disparities in tissue plasminogen activator treatment rate for stroke: A population-based study. Stroke 2011;42:2217-2221.

16. Attenello FJ, Adamczyk P, Wen G, et al. Racial and socioeconomic disparities in access to mechanical revascularization procedures for acute ischemic stroke. J Stroke Cerebrovasc Dis 2014;23:327-334.

17. Cruz-Flores $S$, Rabinstein A, Biller J, et al. Racial-ethnic disparities in stroke care: The American experience: A statement for healthcare professionals from the American Heart Association/American Stroke Association. Stroke 2011;42: 2091-2116.

18. Jacobs BS, Birbeck $G$, Mullard AJ, et al. Quality of hospital care in African American and white patients with ischemic stroke and TIA. Neurology 2006; 66:809-814.

19. Hoffman KM, Trawalter S, Axt JR, Oliver MN. Racial bias in pain assessment and treatment recommendations, and false beliefs about biological differences between blacks and whites. Proc Natl Acad Sci U S A 2016;113:4296-4301.

Address correspondence to: Shaun Ajinkya, MD, MPH

Department of Neurology Medical University of South Carolina 96 Jonathan Lucas Street Room 301, MSC Code: 606 Charleston, SC 29425 USA

E-mail: ajinkya@musc.edu

Received: May 20, 2019

Revised: July 4, 2019

Accepted: July 6, 2019

Online Publication Date: November 22, 2019 